\title{
Crashworthiness characteristics on the Optimal Design of circular stepped tube subjected to axial impact
}

\author{
M.M. AbdElwahab ${ }^{1, a^{*}}$ \\ ${ }^{1}$ Military Technical College, Cairo, Egypt \\ ageneral.abdelwahab@yahoo.com
}

\section{Keywords: Circular, Mechanical Analysis, Optimal Design}

\begin{abstract}
Crashworthiness design draws considerable attention and interest in energy absorption systems. An optimal design of circular stepped tubes based on free inversion is presented in the current work to improve and enhance the crashworthiness characteristics under axial impact loading. Circular tubes have been known to be efficient energy absorbers per unit weight, and may be considered as the main part of energy absorbing devices even for frequently impact operation like landing gear or for sudden accidents like other vehicles. The precise choice of the circular tubes' material, shape, and mechanism may save a person life or save a valuable equipment from damage. Adaptive Multiple-Objective Optimization (AMO) can obtain more light and efficient section. An optimization of variable parameters, which represent the shape of a stepped tube under axial impact, is performed using the platform of ANSYS-DESIGN-EXPLORATION linked with LSDYNA code. The analysis leads to $16 \%$ reducing in mass with corresponding $31 \%$ improving in energy absorption.
\end{abstract}

\section{Introduction}

Crashworthiness is the most important design consideration in the energy absorption applications such as car bumpers, subfloors of aircrafts, the bottom of lifts, train buffers, marine structures, and machinery and so on [1]. The crashworthiness characteristics considered include load-displacement history, total absorbed energy, and specific absorbed energy, stroke-crushing length, mean crushing force and crush force efficiency. Among of them, the specific energy absorption (SEA, energy absorption per unit mass) is one of the most extensive aspects to evaluate the efficiency of energy absorption systems [2-3]

In the recent years, a type of energy absorber known as thin-walled tubes. Thin-walled tubes can be produced with different geometric shapes such as circular, conical, square, taper, octagonal, hexagonal, frusta, S-shaped and so on. Amongst them, thin -walled circular tubes are the most frequently used in energy absorbing devices due to their high strength, high stiffness, low weight, inexpensive, versatile, easy in manufacturing, excellent behaviors on energy absorption characteristics and load-carrying capacity [4]. In spite of, the thin-walled conical tubes have been considered preferable to thin-walled circular tubes as energy absorbers; therefore, it provides a desirable stable crush load-displacement curve response under axial loading and minimize the chances of collapse by global buckling which is an undesirable deformation mode since it reduces the energy absorption capacity [5].

Thin-walled tubes can be classified into different types of deformation mechanisms such as splitting, friction, extrusion, expansion, trigger, ribs, metal cutting and inversion [6-11]. This study concentrates on the inversion mechanism. The inversion process has widely used as energy absorber devices due to its constant inversion load during the process, which obtain an ideal long stable crush load-stroke curve [11]. The thin-walled tube can be inverted inside-out or outside-in. there are two basically ways to produce the inversion tube: the first one is a free inversion and the second is inversion with a die [12-13].

In this paper, a new optimal design of circular stepped tubes based on free inversion is presented to improve and enhance the crashworthiness characteristics under axial impact loading. Adaptive 
Multiple-Objective Optimization (AMO), more light and efficient section can be obtained. An optimization of variable parameters, which represent the shape of a circular stepped tube under axial impact, is performed using the platform of ANSYS-DESIGN-EXPLORATION linked with LSDYNA code.

\section{Methodology}

Structure Configuration. Assuming an axial force F applies on a circular stepped tube with double cylinders, however, the Upper tube (T1) and the Lower tube (T2). There are four variable geometrical parameters as follow: (T1UPR) the upper radius of the upper tube T1, (T1LOR) the Lower radius of the upper tube T1, (STEPWIDTH) the horizontal step length between the two tubes and, (T2LOR) the lower radius of the lower tube T2. Moreover, the other fixed parameters are the vertical height of the both tubes (T1Height, T2Height) and the whole thickness as shown in Fig. 1.

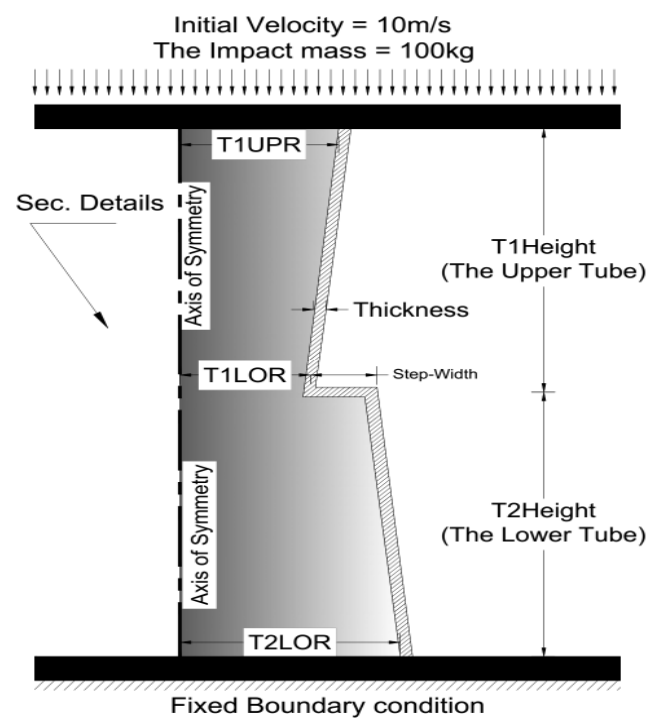

Fig. 1 The schematic drawing shows the Upper tube (T1) and the Lower tube (T2) parameters.

Crashworthiness Criteria for Energy Absorber. Several authors to assess the performance and efficiency of the energy absorber [1-3], in this study the desired parameters, which used for comparison and could be obtained from load-displacement curve as follows, have presented many parameters used to judge the crushing response of structural members, these parameters:

Total absorbed energy (EA). The total absorbed energy during axial crushing is calculated by the area under load-displacement curve from the following equation:

$$
E_{\text {abserbed }}=\int F d \delta
$$

where, $\mathrm{F}$ and $\delta$ are crushing load and crushing distance, respectively.

Stroke Length (SL). When an absorber is crushed to a certain crushing distance (Stroke Length $\mathrm{SL}$ ), the structure in "densification zone" and the corresponding force level reaches very large and unpractical values.

Finite Element Modelling Data. The explicit non-linear finite element code ANSYS-DESIGNEXPLORATION linked with LSDYNA code [14] is used to optimize the effect of shape parameter on energy absorption characteristics of aluminum circular stepped tube subjected to axial impact crushing. The sidewall of tubes is modeled with four-node shell elements with five integration points through the thickness and one integration point in the element plane. The bottom-stepped tube is fixed to a stationary rigid plane, a rigid body with an initial impact velocity of $10 \mathrm{~m} / \mathrm{s}$ strikes the circular stepped tube axially and the mass of the rigid body is $100 \mathrm{~kg}$ as shown in Fig. 3 . Assume that the friction coefficient between all surfaces in contact is 0.25 . All models are made of aluminum al-loyAA6060-T4 with the following mechanical properties: Young's Modulus E=68.2 
$\mathrm{GPa}$, initial yield stress $\sigma y=80 \mathrm{MPa}$, the ultimate stress $\sigma \mathrm{u}=173 \mathrm{MPa}$, Poisson's ratio $v=0.3$, power law exponent $n=0.23$ and the density $\rho=2770 \mathrm{~kg} / \mathrm{m} 3$ [15]. The material properties are set to be constant for all stepped tubes.

Optimization Objectives. The optimization has two direct objectives the first is minimum mass with maximum Energy Absorption (EA), moreover one indirect objective, which is maximum Inversion Stroke Length (ISL). The way to achieve this indirect objective depends on its definition so that; the objective is the maximum of corresponding distance parameter for maximum force below $150 \mathrm{~mm}$ distance to be maximum. For more explanations, with some changes in the tubes geometrical shape the section can reach the densification zone after longer stroke length.

In addition, the optimization has two important constrains; however, these constrains are important for the inversion mechanism. The two constrains are to keep the force at distance $50 \mathrm{~mm}$ and the force at distance $100 \mathrm{~mm}$ below $19 \mathrm{KN}$, however without these two constrains the algorithm will be governed to choose the buckling mechanism instead of inversion mechanism as max. Energy Absorption is selected as a direct objective.

Adaptive Multiple-Objective Optimization (AMO) Workflow. A mathematical optimization combines a Kriging response surface and the Multi-Objective Genetic Algorithm (MOGA) optimization algorithm as shown in Fig. 2, which presents the algorithm workflow.

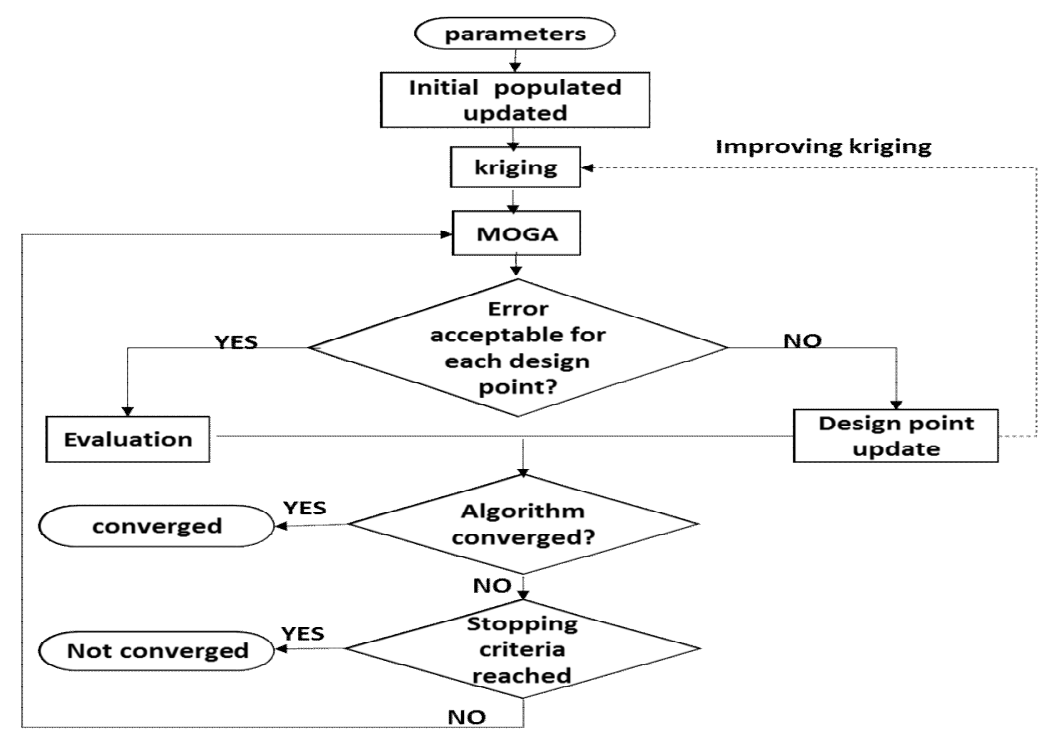

Fig. 2 Adaptive Multiple-Objective Optimization Workflow [14].

It allows either generating a new sample set or using an existing set. Except when necessary, the optimizer does not evaluate all design points. The general optimization approach is the same MOGA, but a Kriging response surface is used; part of the population is "simulated" by evaluations of the Kriging and the Kriging error predictor reduces the number of evaluations used in finding the first Pareto front solutions [14].

\section{Results and Discussion}

Theoretical Prediction Comparison. Recalling Colokoglu and Reddy [9] equation for the initial inversion tube to calculate $\left(\mathrm{P}_{\mathrm{s}}\right)$ for a free inversion tube given by:

$$
P_{s}=2 \pi 6_{o} r t\left(\frac{b}{r}+\frac{t}{4 b}\right)
$$

where, Ps the steady inversion load, $\mathrm{r}$ is the radius of the tube and $\mathrm{b}$ the knuckle radius and $\mathrm{b}$ is set to be $\sqrt{r t} / 2$.

By substituting in equation no. (2), which can be used only for the initial configuration, therefore, its cylindrical shape, so Ps $=9.54 \mathrm{KN}$, and recalling the dynamic factor range concluded by Simhachalam Srinivas [16]. For Aluminum alloys AA6060 T4 under different strain rates, thus for 
$10 \mathrm{~m} / \mathrm{s}$ concludes the range from 1.1 to 1.13 , so (Ps) will be from $10.5 \mathrm{KN}$ to $10.78 \mathrm{KN}$ which agree with an error plus $4.8 \%$ compared with the FEM analysis results in $11.3 \mathrm{KN}$.

The optimized stepped tube results comparison. It is very clear in Fig. 3 that the stepped tube deformation can be divided into three main stage, however, the first stage is the curling (inversing) stage with its famous steady line in the curve, the second stage is the buckling stage with its famous fluctuated curve (starts at $100 \mathrm{~mm}$ in the initial tube curve), and the third stage is the densification stage wherever the force increases with unpractical values because the section has no availability to deform more.

Table 1. Input/output geometric parameters for the stepped tubes.

\begin{tabular}{|c|c|c|c|c|c|}
\hline Parameters & $\begin{array}{c}\text { T1UPR } \\
\mathrm{mm}\end{array}$ & $\begin{array}{c}\text { T1LOR } \\
\mathrm{mm}\end{array}$ & $\begin{array}{c}\text { T2UPR } \\
\mathrm{mm}\end{array}$ & $\begin{array}{c}\text { T2LOR } \\
\mathrm{mm}\end{array}$ & $\begin{array}{c}\text { Step-Width } \\
\mathrm{mm}\end{array}$ \\
\hline $\begin{array}{c}\text { Initial Stepped } \\
\text { Tube }\end{array}$ & 18 & 18 & 26 & 26 & 8 \\
\hline $\begin{array}{c}\text { Optimized } \\
\text { Stepped Tube }\end{array}$ & 17.9 & 16.1 & 24.7 & 16.9 & 8.6 \\
\hline $\begin{array}{c}\text { Variation } \\
\text { Range }\end{array}$ & $15-25$ & $15-25$ & $20-35$ & $15-25$ & $5-10$ \\
\hline
\end{tabular}

Table 2. Numerical results for objective parameters.

\begin{tabular}{|c|c|c|c|}
\hline Objective & Mass kg & $\begin{array}{c}\text { Inversion } \\
\text { (EA) KJ }\end{array}$ & $\begin{array}{c}\text { Total (EA) } \\
\text { KJ }\end{array}$ \\
\hline $\begin{array}{c}\text { Initial } \\
\text { Stepped Tube }\end{array}$ & 0.1592 & 0.0841 & 1.58 \\
\hline $\begin{array}{c}\text { Optimized } \\
\text { Stepped Tube }\end{array}$ & 0.1379 & 0.0966 & 2.08 \\
\hline $\begin{array}{c}\text { Improvement } \\
\text { Percentage }\end{array}$ & $-16 \%$ & $15 \%$ & $31 \%$ \\
\hline
\end{tabular}

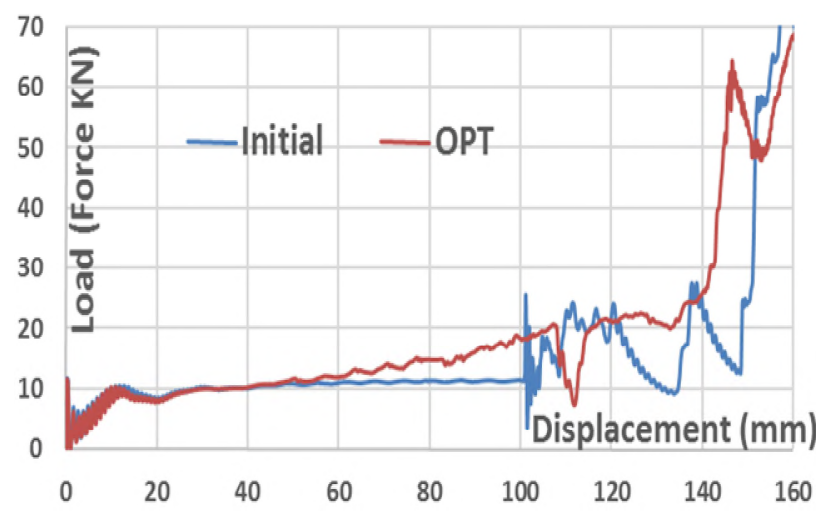

Fig. 3 Load-displacement curve of the initial tube and optimized tube.
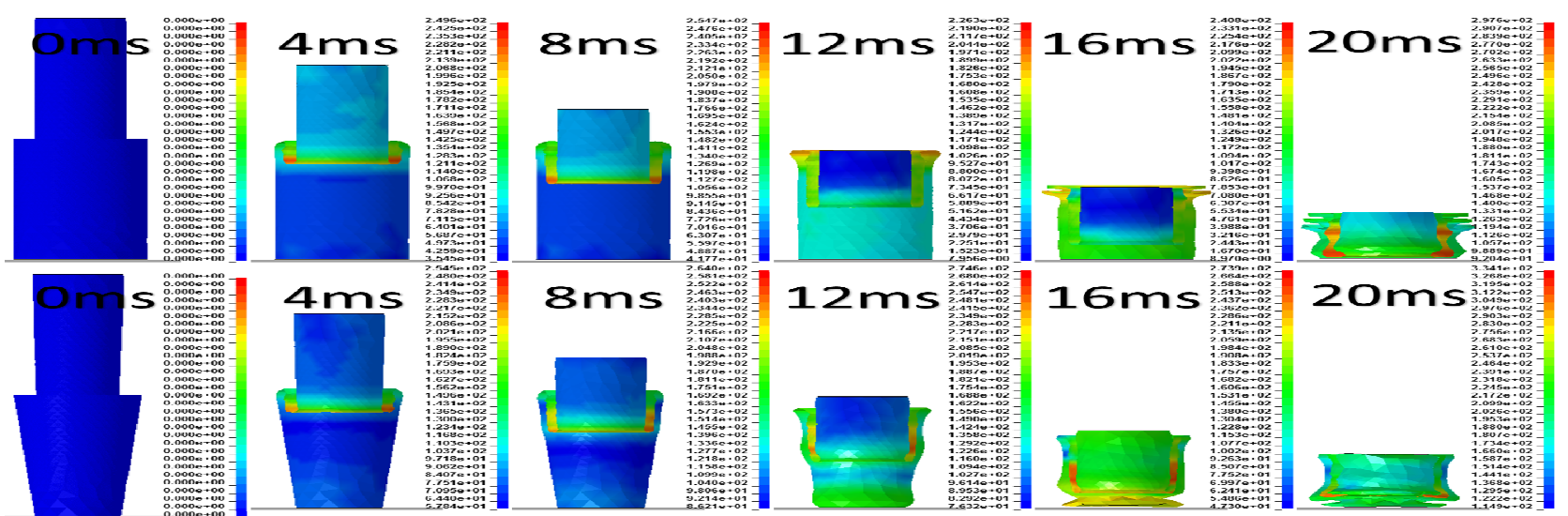

Fig. 4 Successive shots show the deformed shape with Von-Mises stress contours.

(The upper shots for the initial tube and, the lower shots for the optimized tube).

As shown in Table 1 and, Table 2 the new conical stepped tube which is generated from the optimization algorithm improves the energy absorption at the inversion stage by $15 \%$ which shares in the total energy absorption to be improved by $31 \%$, however, the corresponding mass decreases by $16 \%$. An important note is shown clearly in Fig. 4 if a comparison done between the two $12 \mathrm{~ms}$ shots for the both stepped tubes (initial and opt.), however, the height of the lower tube for the initial tube is almost fixed from 0ms, but the height of lower tube in the opt. tube decreases, which means it curls (inverses) a little with the upper tube, furthermore, this curling with a greater value of force, so that the new section improves the curling stage force and deflection values. Physically the cylinder has the same radius all over its height unlike the cone, however, these characteristics influence directly on the densification stage as shown in Fig. 3 at the end of the curves and so in Fig. 4. In $20 \mathrm{~ms}$ shots in the identical deformed humps for the cylinder, therefore, the deformed material 
in the cylinder concentrates on the cylinder circumference unlikely the cone, thus, in the conical case; there is more space between the small circle circumference and the large circle circumference, which gives the defamed material more available space for more available deformation.

Optimization Tradeoffs and Sensitivity Analysis. The AMO algorithm gives the first Pareto front objectives results and their corresponding input parameters as shown in Fig. 5. These results are very helpful to draw a complete image about the multi-objectives relationships.
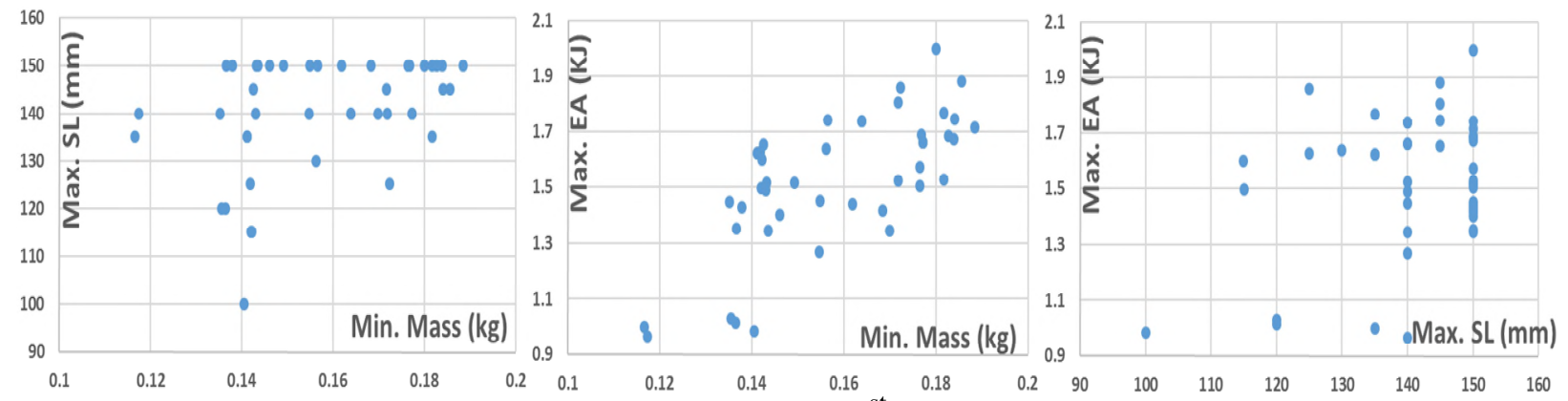

Fig. 5. Tradeoff for $1^{\text {st }}$ Pareto front.

The sensitivity analysis done by AMO algorithm estimates the influence of the input parameters on the objectives ratios as shown in Fig. 6. For a minimum mass objective, all the parameters affect in increasing the mass of their increase but in this problem, T1LOR affects more as it increases the upper and lower tubes diameters while Step-Width is constant as shown in Fig. 1. For maximum Energy Absorption, the most effective parameter is T2LOR therefore, it affects directly on the lower cone stiffness, and however, the lower cone is the responsible part for the buckling stage. For maximum Stroke Length, it is very clear that the ratio between T1LOW and Step-Width controls the curling mechanism, which means this ratio makes the curling of one tube only or both of them, which affects directly on the ISL as shown in Fig. 3.

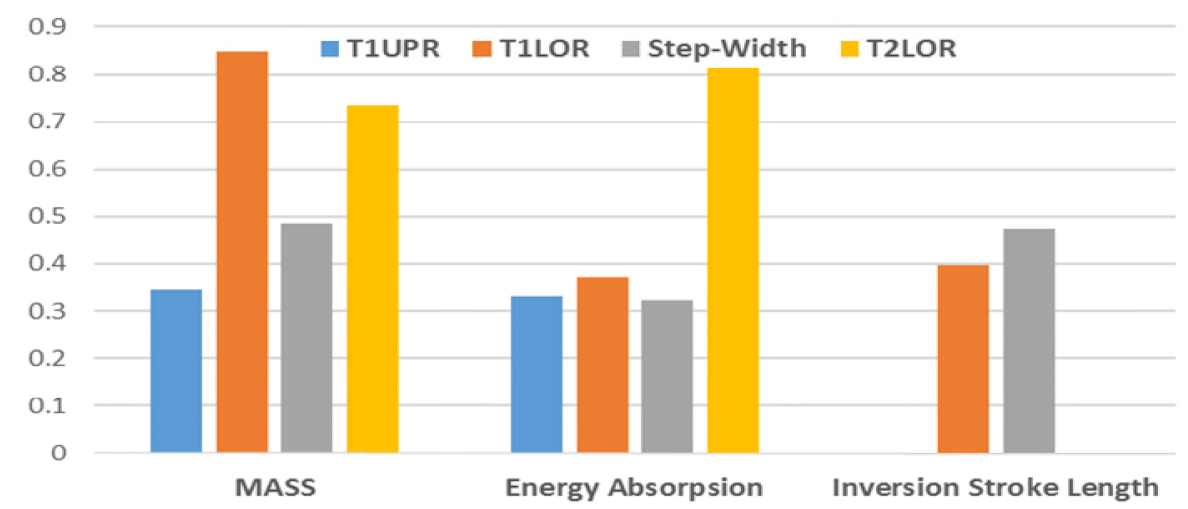

Fig. 6 Input Parameters-Objectives sensitivity analysis chart.

\section{Conclusion}

An optimal design of circular stepped tubes based on free inversion is presented in the current work to improve and enhance the crashworthiness characteristics under axial impact loading. The main findings of this study can be drawn as follows:

1- The explicit LSDYNA model results agree with the theoretical prediction with plus $4.8 \%$ error.

2- The optimization improves inversion EA with $15 \%$ and Total EA $31 \%$ with decreasing the mass with $16 \%$.

3- Conical stepped tubes better than the cylindrical stepped tube in the total stroke length distance, as the conical shape distributes the deformed material within different diameters the small and the large one in the both cones unlikely the concentration of cylinder concentrates in one constant diameter. 
4- The lower radius of the lower conical tube is the most effective parameter on the maximum EA parameter.

5- The difference between two stepped conical tubes radii at the contact zone between them can control the inversion mechanism, which means to make the upper cone curls or the lower cone curls or both of them.

\section{References}

[1] D. F. Shanahan, Basic Principles of Crashworthiness. Pathological Aspects and Associate Biodynamics in Aircraft Accident Investigation, 2004.

[2] G. Lu, T. Yu, Energy absorption of structures and materials, Elsevier, 2003.

[3] N. Jones, Energy-absorbing effectiveness factor. Int. J. Impact Eng. 37(6) (2010) 754-765.

[3] A. G. Olabi, E. Morris, M. Hashmi, Metallic tube type energy absorbers: a synopsis. Thin-walled Struct. 45(7) (2007) 706-726.

[4] A. Alghamdi, Collapsible impact energy absorbers: an overview. Thin-walled Struct. 39(2) (2001) 189-213.

[5] N. Jones, Structural impact. Cambridge university press, 2011.

[6] W. Johnson, S. Reid, Metallic energy dissipating systems. Appl. Mech. Rev. 31(3) (1978) 277-288.

[7] N. Gupta, S. Gupta, Effect of annealing, size and cut-outs on axial collapse behaviour of circular tubes. Int. J. Mech. Sci. 35(7) (1993) 597-613.

[8] A. Niknejad, B. Rezaei, G.H. Liaghat, Empty circular metal tubes in the splitting processtheoretical and experimental studies. Thin-walled Struct. 72 (2013) 48-60.

[9] D. H. Bich, et al. Nonlinear static and dynamic buckling analysis of imperfect eccentrically stiffened functionally graded circular cylindrical thin shells under axial compression. Int. J. Mech. Sci. 74 (2013) 190-200.

[10]M. Shakeri, S. Salehghaffari, R. Mirzaeifar, Expansion of circular tubes by rigid tubes as impact energy absorbers: experimental and theoretical investigation. Int. J. Crashworthiness, 12(5) (2007) 493-501.

[11]J. Yang, et al. Energy absorption of expansion tubes using a conical-cylindrical die: Experiments and numerical simulation. Int. J. Mech. Sci. 52(5) (2010) 716-725.

[12] S. Al-Hassani, W. Johnson, W. Lowe, Characteristics of inversion tubes under axial loading. J. Mech. Eng. Sci. 14(6) (1972) 370-381.

[13]T. Reddy, Guist and Marble revisited-on the natural knuckle radius in tube inversion. Int. J. Mech. Sci. 34(10) (1992) 761-768.

[14]F. Manual, ANSYS, Release 15.0 ANSYS Documentation. ANSYS Inc, Canonsburg, PA,. 2013.

[15] S. P. Santosa, et al. Experimental and numerical studies of foam-filled sections. Int. J. Impact Eng. 24(5) (2000) 509-534.

[16]B. Simhachalam, K. Srinivas, C. L. Rao, Energy absorption characteristics of aluminium alloy AA7XXX and AA6061 tubes subjected to static and dynamic axial load. Int. J. Crashworthiness, 19(2) (2014) 139-152. 